\title{
Focused Ion Beam Based Sample Preparation Techniques
}

\author{
R. M. Langford, A. K. Petford-Long and *P. Gnauck \\ Department of Materials, University of Oxford, United Kingdom, OX1 3PH \\ * LEO Elektronenmikroskopie GmbH, Oberkochen, Germany
}

Focused ion beam (FIB) s ystems are routinely used for the preparation of site specific transmission electron microscopy (TEM) specimens of a diverse range of materials such as semiconductors, ceramics and metals. In this article we report on some of the FIB sample preparation methods that we have developed or are developing to improve upon or extend the current FIB sample preparation techniques.

The 'trench' and the 'lift-out' techniques can be used for the preparation of plan-view specimens. A specimen is cleaved/or polished and mounted in the FIB system with the cleaved edge vertical. The plan -view specimens are then made at the cleaved surface using either one of the two techniques. A limitation with this approach is that because it is not easy to cleave or polish a sample close to a feature of interest, it is difficult to use it to make site specific plan-view specimens. The approach we use to make such specimens is to FIB mill a wedge shaped piece of material which is free from the substrate. (Three cuts are made in the sha pe of a ' $U$ ' and a fourth is made at $45^{\circ}$ such that it cuts through the bases of the other three). A micromanipulator and glass needle are used to lift the wedge out from the substrate and to orientate it with the surface of interest vertical, as shown in Figure 1. The plan-view specimen is then made from the wedge using the lift-out technique.

For some samples, such as crack tips, the region of interest can be more than $20-30 \mu \mathrm{m}$ beneath the surface and therefore the required FIB milling time is prohibiti vely long. To reduce the required FIB milling time we have used broad ion beam (BIB) milling on the top surface of the sample to decrease the distance to the feature of interest ${ }^{(2)}$. A slice containing the crack tip is mounted onto a copper disc and then BIB milled as shown in Figure 2. It is repeatedly examined using a light microscope to determine the distance of the feature of interest to the surface of the sample. Once the distance has been reduced to around $5 \mu \mathrm{m}$ the specimen is mounted in the FIB system with the support grid vertical and the TEM sample is made using the trench technique. Another advantage of this approach is that the BIB milling also reduces the width of the slice, and therefore the lengths of the trenches, which reduces the degree of geometric shadowing when imaging in the TEM.

FIB milling using a $30 \mathrm{keV}$ ion beam can introduce a considerable amount of damage at the sidewalls of the specimen which can affect high resolution imaging. Low energy $(5 \mathrm{keV})$ milling with the sample tilted by $10^{\circ}$ reduces the amount of damage but may result in preferential milling of the different materials or layers. The approach we use to reduce both the thickness of the specimen and depth of the damage ${ }^{(3)}$ is to prepare the specimen using the lift-out technique and to place it onto a $0.5 \mu \mathrm{m}$ thick silicon support membrane into which an array of windows has been patterned. The top side of the specimen is sputtered in a BIB system using a low incident energy $(3 \mathrm{keV})$ and shallow angle $\left(2-3^{\circ}\right)$. The specimen is then t urned over using a glass needle and micromanipulator so that the other side can be sputtered.

Automatic preparation of a specimen considerably increases the utilization of the FIB system, enabling it to be run over night. We are currently developing a procedure for the automatic thinning of specimens which warp during the final thinning stages due to intrinsic stress. Relaxation of the specimen in the ion beam can result in non-uniform thinning and also in the milling of the features of 
interest. During the line milling/cleaning stage, the intensity of the secondary electron signal is monitored and if it dramatically increases the thinning is stopped. An image of the specimen is taken and the positions of its edges, relative to FIB milled alignment marks, are determined. The FIB is then scanned along the edges of the specimen to further thin it uniformly. This method is shown diagrammatically in Figure 4. It is realised that this approach will not suitable for regions of the specimen which warp into the plane perpendicular to the ion beam but is suitable for thin films at the top of the specimens.

\section{References}

[1] R. M. Langford et al., J. Vac. Sci. Tech. A 19 (2001) 982

[2] S. Lozano-Perez et al, Inst. Phys. Conf. Ser. No. 168 (2001) 191

[3] R. M. Langford et al. J. Vac. Sci. Tech., B 19 (2001) 755

[4] The authors gratefully acknowledge the support of EPSRC

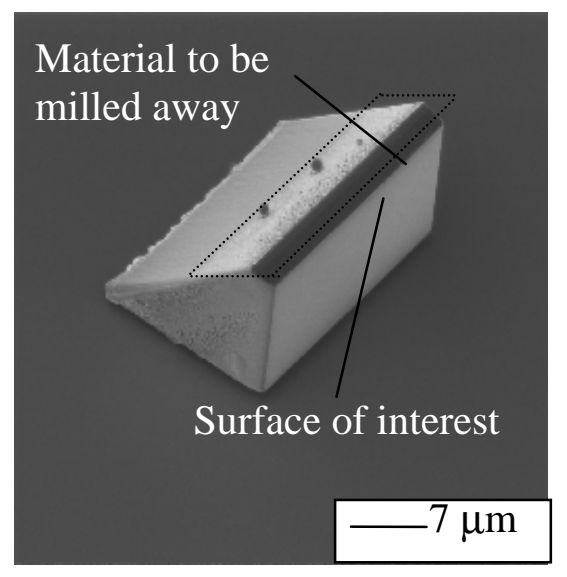

Fig. 1. FIB milled wedge orientated on the substrate with the surface vertical.

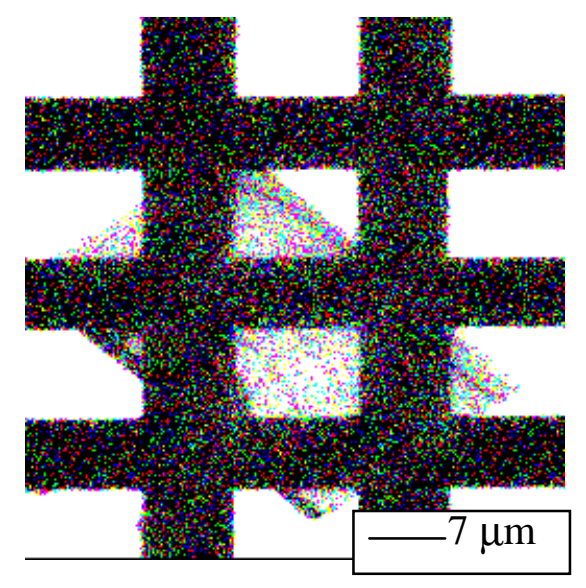

Fig. 3. TEM specimen on Si support membrane for BIB milling and imaging.

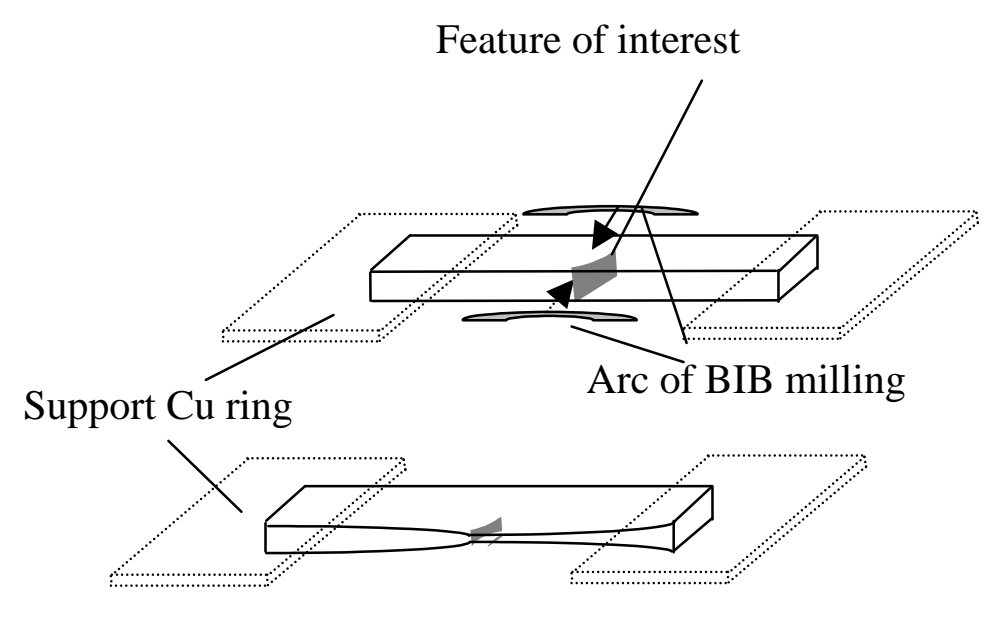

Fig. 2. BIB milling of the slice

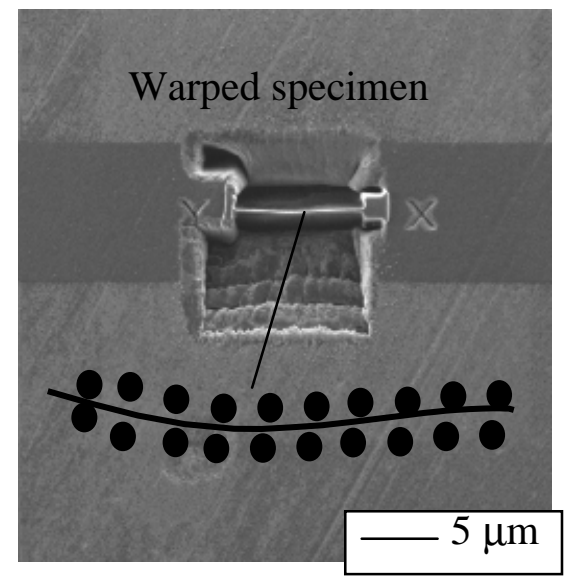

Fig. 4. Software determines the edges of the warped specimen so that the ion beam can be scanned along its edges for further thinning 